\title{
Value of Mastoidectomy in Malignant Otitis External
}

\section{Original Article}

\author{
Ossama Mustafa Mady, Ahmed Abdelmoneim Teaima, Michael Rezk Shafek \\ Otorhinolaryngology, Ain Shams University Faculty of Medicine, Cairo, Egypt
}

\begin{abstract}
Objectives: To define the value of mastoidectomy in cases of malignant otitis externa. Study design: Case Series.

Patients and Methods: Study of Patients with malignant otitis externa (MOE) treated by mastoidectomy from Jan 2016 to October 2018 and followed up clinically, laboratory and radiologically.

Results: Postsurgical siginifacant resolution of symptoms and regression of the disease. Otalgia showed siginificant decrease by assessment with Visual Analogue Score. Also there are improvement of otorrhea, decrease of the reactive indicators as Erythrocyte sedimentation rate (ESR) and C-reactive protein (CRP) and radiological improvement of osteomyelitis in Computed Tomography and technetium-99m methylene diphosphonate (MDP) and gallium-67 citrate scintigraphy.

Conclusion: In patients with MOE, resistant to medical treatment and have evidence of mastoiditis and Middle ear affection, cortical mastoidectomy may be the treatment for both symptom relieve and regression of the disease.
\end{abstract}

Key Words: Malignant otitis externa, otalgia, otitis externa, otorrhea.

Received: 20 September 2019, Accepted: 22 November 2019

Corresponding Author: Ossama M. Mady, MD, Lecturer of Otorhinolaryngology Department. Faculty of Medicine. Ain Shams University. Egypt, Tel.: 01117341201, E-mail: omady84@gmail.com

ISSN: 2090-0740, July 2020 Vol.21, No.2

\section{INTRODUCTION}

Malignant otitis externa (MOE) is a progressive disease. It is an aggressive form of skull base osteomyelitis. Chandler first described MOE in 1968. Since then different criteria of diagnosis were settled and also different forms of treatment ${ }^{[1]}$. Although mortality from this disease decreased dramatically in the past few decades due to advances in antibiotics, it still forms challenge for otologists due to its impact in the patient life especially associated otalgia ${ }^{[2]}$. Its treatment mainly depends on control of general disease and management of local disease medically. However, surgery may have a role and may be the main one in some cases. So here we present our experience with surgical treatment and the criteria to choose it in retrospective study from our tertiary referral hospital.

\section{METHODOLOGY:}

Data We reviewed MOE patients in the last three years from 2016 till end of 2018. Diagnosis was done using Cohen criteria and its modifications ${ }^{[3,4]}$. Then we chose the patients underwent surgery for MOE to be included in our study. The total number of patients with MOE admitted to our hospital in this period were seventy six, only thirty two patients underwent surgery, three patients lost during follow up and one patient died with myocardial infarction, so twenty eight patients complete our study and follow up.

\section{Inclusion criteria of surgical patients:}

- Patients with MOE, clinically with odematous tender external auditory canal with or without granulation tissue in external canal (Figure 1).

- No improvement on medical treatment in the form of local and systemic antibiotics according to culture for at least 6 weeks, and control of general condition and diabetes.

- Also no improvement on hyperparic oxygen therapy for 20 sessions.

- Radiological evidence of bone involvement and mastoiditis in CT scan temporal bone (Figure 2, 3).

- $\quad$ Elevated ESR and CRP. 


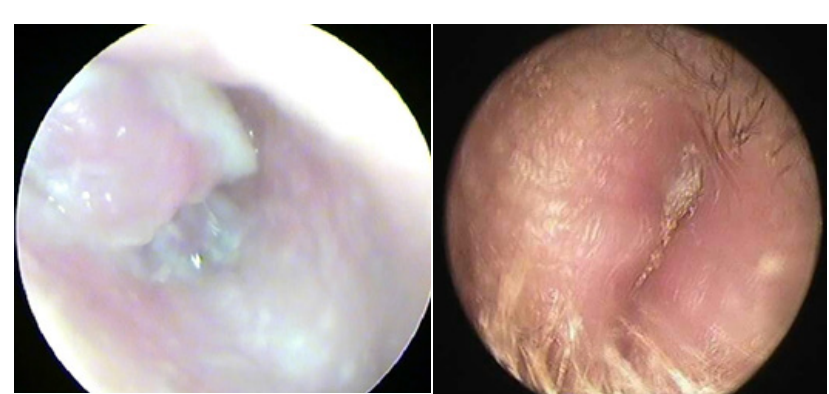

Fig. 1: Endoscopic examination of external auditory canal in MOE patients

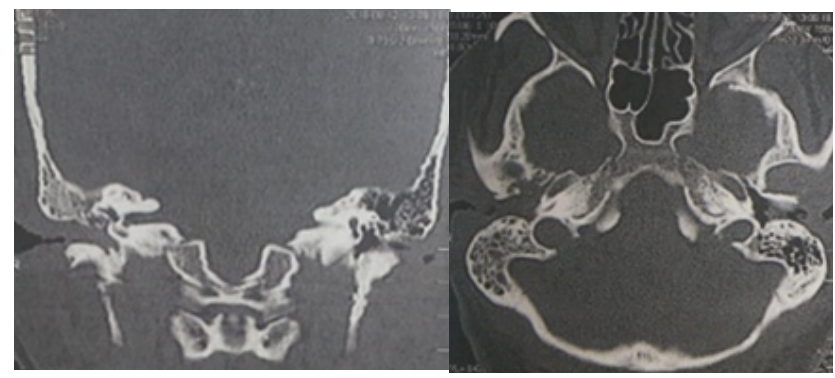

Fig. 2: Right sided mastoiditis and skull base osteomyelitis in patients with MOE

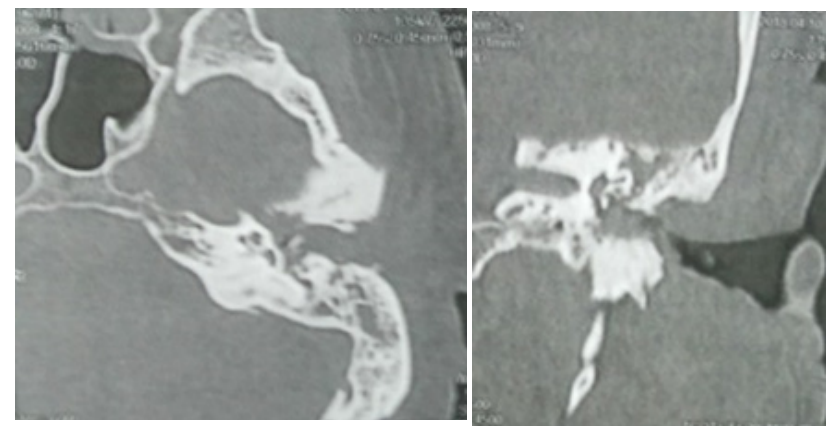

Fig. 3: Left sided mastoiditis and skull base osteomyelitis in patients with MOE

Table 1: Results of culture

\begin{tabular}{llllll} 
Organism & Candida & Klebsiella & MRSA & Pseudomomas & Staph \\
\hline no & 1 & 2 & 4 & 16 & 5 \\
percentage & $4 \%$ & $7 \%$ & $14 \%$ & $57 \%$ & $18 \%$ \\
\hline
\end{tabular}

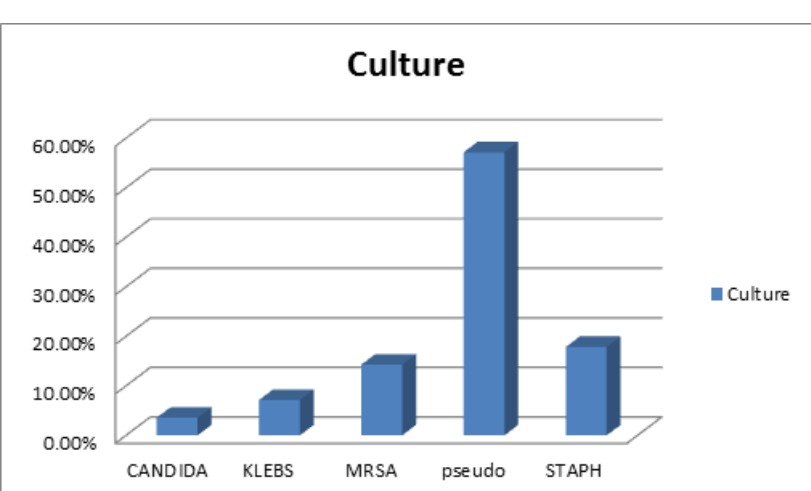

Fig. 4: Culture Results
Twenty eight patients complete our study, Patient mean age 58 years old (SD 5.2), seventeen are male (61\%) and eleven are females (49\%), pre and postoperative otalgia were assessd by visual analogue scale, regarding postoperative otorrhea was compared to the preoperative as a percentage. Temporal bone affection and osteomyelitis in CT were classified according to Chin et al radiological grading, eleven patients of our study were grade II (with bone involvement limited to the Mastoid), thirteen patients were grade III (MOE extending medially to involve the petrous temporal Bone), four patients were grade IV (MOE extending medially to involve the petrous apex or with cranial nerve involvement or anteriorly to involve the facial bones, posteriorly to involve the occipital bone, or spread to the contralateral base of skull $)^{[2]}$.

Culture and sensitivity of the discharge was done and revealed sixteen patients had pseudomonas in the discharge, five with methicillin sensitive staph aureus, four with methicillin rensitive staph aureus (MRSA), two with klebsiella and one with candida (Figure $4 \&$ Table 1 ). They received intravenous antibiotics according to cultures (ciprofloxacin, tazocin, ceftazidine, ceftriaxone, vancomycin, meronam) and local treatment in the form of ear drops and packing and debridement. Strict control of diabetes was done. Total period of hospitalization and abs therapy around 6 weeks. Although all these measures no improvement occurred.

So the decision was made to go for surgery. During surgery, first we do canal debridement, polypectomy if present (four patients with aural polyp), then cortical mastoidectomy. Cortical mastoidectomy is done through postauricular incision going down with this incision directly to the mastoid bone without any tissue dissection. We take care not to elevate of the tympanomeatal flap (Posterior meatal wall and tympanic membrane) to decrease spread of infection. Then we start drilling of the mastoid as usual but take care to open all mastoid air cells including tip, zygomatic root, retrosigmoid air cells. If needed we can also do myringotomy to maximize the aeration of the middle ear and the mastoid. We try to make the surgery time as short as possible to avoid prolonged surgery time in these high risky patients. In all these patients, mastoid was unhealthy and signs of chronic mastoiditis were always there. During closure of the incision, we suture it by horizontal mattress suture in one layer as we do the incision as one layer to the bone. Then these patients continue on medical treatment according to culture and sensitivity as presurgical and we assess the response clinically and by laboratory indicators after two weeks and then by imaging after three months.

\section{RESULTS}

We followed patients clinically, by laboratory indicators and by imaging. Clinical improvement started from the first week and we make the assessment clinically and laboratory by two weeks which showed significant improvement. Most patients (25 patients) discharged on oral medical treatment 
after two weeks and even stopped it after ten days. Three patients needed prolonged hospitalization due to associated cardic and metabolic problems. Radiological follow up after three months revealed significant improvement of osteomyelitis in CT scans and technetium-99m methylene diphosphonate (MDP) and gallium-67 citrate scintigraphy.

\section{Patients History and Clinical Signs:}

Regarding otalgia which were the major complain of the patients, pre-operative all the study group had severe pain, post operative follow up by visual analogue scale (VAS) showing significant improvement of otalgia. We used VAS to evaluate persistent, severe otalgia; 10 points for the worst imaginable pain and 0 points for no pain. Regarding otorrhea during follow up, significant decrease in percent of otorrhea as shown in (Table $2 \& 3$ ).

Six patients of our study group also had facial nerve palsy (four complete and two partial LMNFP) during follow-up no improvement of facial paralysis.

Table 2: Improvement of otalgia and otorrhea two weeks postoperatively

\begin{tabular}{lllll}
\hline & Mean & SD & P value & Sig \\
\hline otalgia preoperative & 10 & 0 & 0.001 & HS \\
otalgia postoperative & 3.9 & 1.8 & & 0.001 \\
otorrhea preoperative & 100 & 0 & & \\
otorrhea postoperative & 43 & 18.38 & & \\
\hline
\end{tabular}

Table 3: Patients involved in the research with their results

\begin{tabular}{|c|c|c|c|c|c|c|}
\hline No of Patients & Age & Gender & $\mathrm{HbA1c}$ & Culture & $\begin{array}{l}\text { otalgia post } \\
\text { operative }\end{array}$ & $\begin{array}{l}\text { otorrhea post operative } \\
\text { (percentage) }\end{array}$ \\
\hline 1 & 55 & $\mathrm{M}$ & 7.4 & Staph aureus & 5 & 50 \\
\hline 2 & 58 & $\mathrm{~F}$ & 6.5 & Pseudomonas & 2 & 50 \\
\hline 3 & 60 & $\mathrm{~F}$ & 7 & Pseudomonas & 2 & 70 \\
\hline 4 & 57 & M & 6.6 & MRSA & 3 & 40 \\
\hline 5 & 45 & $\mathrm{~F}$ & 7.2 & Pseudomonas & 2 & 20 \\
\hline 6 & 60 & $\mathrm{~F}$ & 6.9 & Staph aureus & 5 & 20 \\
\hline 7 & 57 & M & 6.7 & Pseudomonas & 3 & 70 \\
\hline 8 & 62 & M & 6.8 & Klebsiella & 2 & 20 \\
\hline 9 & 58 & $\mathrm{~F}$ & 6.5 & Pseudomonas & 2 & 30 \\
\hline 10 & 56 & M & - & MRSA & 3 & 20 \\
\hline 11 & 56 & M & 6.5 & Pseudomonas & 2 & 70 \\
\hline 12 & 49 & M & 6.7 & Candida & 8 & 20 \\
\hline 13 & 62 & $\mathrm{~F}$ & 6.2 & Pseudomonas & 5 & 30 \\
\hline 14 & 68 & $\mathrm{~F}$ & 6.8 & Pseudomonas & 2 & 70 \\
\hline 15 & 57 & M & 6.7 & Staph aureus & 7 & 30 \\
\hline 16 & 58 & M & 6.5 & Pseudomonas & 6 & 60 \\
\hline 17 & 62 & M & - & Staph aureus & 3 & 30 \\
\hline 18 & 58 & $\mathrm{~F}$ & 7 & Pseudomonas & 7 & 70 \\
\hline 19 & 62 & M & 6.5 & Klebsiella & 2 & 40 \\
\hline 20 & 64 & M & 6.4 & Pseudomonas & 3 & 40 \\
\hline 21 & 55 & M & 6.5 & Pseudomonas & 4 & 60 \\
\hline 22 & 57 & $\mathrm{~F}$ & 6.7 & Pseudomonas & 7 & 40 \\
\hline 23 & 55 & $\mathrm{~F}$ & 6.7 & Staph aureus & 5 & 30 \\
\hline 24 & 68 & M & 6.8 & MRSA & 4 & 60 \\
\hline 25 & 54 & $\mathrm{~F}$ & 7.1 & MRSA & 2 & 20 \\
\hline 26 & 69 & M & 6.8 & Pseudomonas & 3 & 40 \\
\hline 27 & 55 & M & - & Pseudomonas & 5 & 60 \\
\hline 28 & 57 & $\mathrm{M}$ & 6.8 & Pseudomonas & 4 & 30 \\
\hline
\end{tabular}


Table 4: Laboratory Finding preoperative and two weeks postoperative.

\begin{tabular}{|c|c|c|c|c|}
\hline No of Patients & ESR Preoperative & ESR postoperative & CRP preoperative & CRP postoperative \\
\hline 1 & 77 & 25 & 18 & 3.6 \\
\hline 2 & 84 & 22 & 22.4 & 5 \\
\hline 3 & 82 & 18 & 10.6 & 4.8 \\
\hline 4 & 89 & 21 & 37.6 & 3.8 \\
\hline 5 & 97 & 22 & 40 & 5.7 \\
\hline 6 & 95 & 24 & 19,5 & 4.7 \\
\hline 7 & 67 & 16 & 27.5 & 7 \\
\hline 8 & 87 & 20 & 22.5 & 5.2 \\
\hline 9 & 84 & 22 & 25.8 & 6 \\
\hline 10 & 93 & 20 & 18 & 3.4 \\
\hline 11 & 92 & 17 & 26.6 & 2.8 \\
\hline 12 & 83 & 23 & 46.8 & 6.8 \\
\hline 13 & 86 & 20 & 18,6 & 4.5 \\
\hline 14 & 63 & 19 & 22 & 5 \\
\hline 15 & 85 & 27 & 23.3 & 4.7 \\
\hline 16 & 79 & 22 & 27.5 & 3.3 \\
\hline 17 & 84 & 20 & 31.6 & 5.8 \\
\hline 18 & 75 & 18 & 17 & 4 \\
\hline 19 & 97 & 25 & 22.3 & 5.4 \\
\hline 20 & 84 & 20 & 29 & 3 \\
\hline 21 & 65 & 22 & 19 & 4.7 \\
\hline 22 & 83 & 26 & 24.4 & 2.8 \\
\hline 23 & 77 & 18 & 62.8 & 6.3 \\
\hline 24 & 93 & 21 & 18.2 & $4 . .7$ \\
\hline 25 & 79 & 19 & 25.8 & 6.8 \\
\hline 26 & 88 & 20 & 33 & 3.6 \\
\hline 27 & 83 & 24 & 23.5 & 6 \\
\hline 28 & 95 & 16 & 27.6 & 5.8 \\
\hline
\end{tabular}

\section{Laboratory Findings}

Pre operative ESR and CRP were elevated. The mean pre operative ESR were 83.7 and post operative 20.9 . The mean CRP preoperative was 26.4. Follow-up CRP returned to reference intervals postoperatively (Table 4).

\section{Radiologic Findings}

Improvement of osteomyelitis and bone affection in CT grading system, but the most important in follow-up was the gallium scan that improved in all patients and return to normal in twenty three patients.

\section{DISCUSSION}

MOE is a progressive osteomyelitis of the temporal bone, usually begin as a simple or benign otitis externa but in patients with diabetes mellitus or immunodeficiency, inflammation may extend to the underlying bone. The infection begins in the dermal and epidermal tissue at the junction of the cartilaginous and osseous EAC and spreads through the Santorini fissures, medially to the tympanomastoid fissure, petrous apex, and skull base bone; inferiorly to the stylomastoid foramen, infratemporal fossa, and neck; posteriorly to the mastoid bone and sigmoid sinus; and anteriorly to the parotid gland and surrounding tissues $^{[5]}$.

The main treatment of MOE is the medical treatment and recently mortality decrease with the advances in antimicrobial therapy. There is an increasing use of surgical management in severe cases of $\mathrm{MOE}^{[1]}$. Our center is a tertiary referral center, most cases referred to the center is MOE cases resistant to the usual medical treatment. In our study pseudomonas was the most common organism in culture (57\%), patients received 
antibiotics according to culture with the other lines of medical treatment, forty four patients of total seventy six patients $(58 \%)$ respond to treatment.

So in the rest of patients, we chose to proceed for surgical intervention specially in the presence of mastoiditis and middle ear affection and resistant otalgia. Our aim is surgical drainage of inflammation and aeration of the temporal bone and mastoid. The results of our study were supported by combined clinical, laboratory and radiological assessment of the patients. During follow-up postoperatively, there were significant decrease in reactive inflammatory indicators (ESR-CRP) and significant improvement in gallium scan, indicating decrease in inflammation that occurred with areation of the mastoid by cortical mastoidectomy. Decrase in inflammation leads to clinical improvement and decrease of otalgia and otorrhea.

We try to decrease the bulk of inflammatory process and to well aerate the ear and it seems to work well by symptoms, laboratory and imaging improving the prognosis of disease

There is increasing trend toward surgical intervention in cases of $\mathrm{MOE}^{[1]}$. In our study, surgically treated patients are about $42 \%$ of all MOE (3276/). Sharma et al mentioned that surgical intervention is about $25 \%$ in $\mathrm{MOE}^{[6]}$. According to Gruber et al, 4 of 5 patients with resistant inflammation need surgery ${ }^{[7]}$. In $\mathrm{U} \mathrm{K}$ astudy was done in Milton Keynes university hospital about MOE, the role of surgery was about $43 \%$ but extent of surgery varying from biobsy, debulking or mastoidectomy ${ }^{[8]}$.

Based on our study, we should consider wide cortical mastoidectomy as a line of treatment in resistant cases of MOE.

\section{CONCLUSION}

In patients with MOE, resistant to medical treatment and have evidence of mastoiditis and ME affection, cortical mastoidectomy may be the treatment for both symptom relieve and regression of the disease.

\section{CONFLICT OF INTEREST}

There are no conflicts of interest.

\section{ACKNOWLEDGEMENT}

Many thanks to our late Professor Hassan Wahba who tailored this technique of surgery and was the main power for this study.

\section{REFERENCES}

1. Hatch JL, Bauschard MJ, Nguyen SA, Lambert PR, Meyer TA, McRackan TR. Malignant Otitis Externa Outcomes: A Study of the University Health System Consortium Database. Ann Otol Rhinol Laryngol. 2018 Aug;127(8):514-520.

2. Chin R, Roche P, Sigston E, Valance N. Malignant otitis externa: an Australian case series. Surgeon. 2012 Oct; 10(5):273-7.

3. Cohen D, Friedman P. The diagnostic criteria of malignant external otitis. J Laryngol Otol. 1987;101:216-221.

4. Mehrotra P, Elbadawey MR, Zammit-Maempel I. Spectrum of radiological appearances of necrotising external otitis: a pictorial review. J Laryngol Otol. 2011;125:1109-1115.

5. Verim A1, Naiboğlu B, Karaca Ç, Seneldir L, Külekçi S, Oysu Ç. Clinical outcome parameters for necrotizing otitis externa. Otol Neurotol. 2014 Feb;35(2):371-6.

6. Sharma S, Corrah T, Singh A. Management of Necrotizing Otitis Externa: Our Experience with Forty-Three Patients. J Int Adv Otol. 2017;13(3): 394-398.

7. Gruber M, Sela E, Doweck I, Roitman A, Uri N, Srouji S, Cohen-Kerem R. The role of surgery in necrotizing otitis externa. Ear Nose Throat J. 2017 Jan; 96(1):E16-E21.

8. Gaurav Chawdhary, Miran Pankhania, Susan Douglas and Ian Bottrill. Current management of necrotising otitis externa in the UK: survey of 221 UK otolaryngologists. Acta Oto-Laryngologica, 2017. http://dx.doi.org/10.1080/00016489.2017.1295468. 